\title{
INTEGRAÇÃO TECNOLÓGICA NA EDUCAÇÃO BÁSICA PÚBLICA BRASILEIRA: UMA ANÁLISE SOBRE A EVOLUÇÃO TEMPORAL E A INTERDISCIPLINARIDADE DO TEMA.
}

\author{
INTEGRACIÓN TECNOLÓGICA EN LA EDUCACIÓN BÁSICA PÚBLICA \\ BRASILEÑA: UN ANÁLISIS SOBRE LA EVOLUCIÓN TEMPORAL Y LA \\ INTERDISCIPLINARIDAD DEL TEMA
}

\author{
TECHNOLOGICAL INTEGRATION IN BRAZILIAN PUBLIC BASIC \\ EDUCATION: AN ANALYSIS OF THE TEMPORAL EVOLUTION AND THE \\ INTERDISCIPLINARITY OF THE THEME
}

\author{
Priscila Cadorin NICOLETE ${ }^{1}$ \\ Simone Meister Sommer BILESSIMO ${ }^{2}$ \\ Patricia de Sa FREIRE ${ }^{3}$ \\ Juarez Bento da SILVA ${ }^{4}$ \\ Marta Adriana da Silva CRISTIANO 5
}

RESUMO: A complexidade inerente ao contexto de integração tecnológica na educação básica - na realidade atual dos estudantes e na cultura digital que permeia a sociedade atual - caracteriza uma transformação na dinâmica da construção do conhecimento, do qual os processos de ensino e aprendizagem, utilizando métodos do ensino tradicional, apresentam dificuldades para conectar-se ao aluno e envolve-lo de forma plena nesse processo. Esse novo contexto passa a exigir pesquisas interdisciplinares para sua melhor compreensão, envolvendo especialistas na área de ciências sociais e áreas mais técnicas como engenharias e ciência da computação. Para responder a questão de como se processa a evolução dos estudos teóricos e empíricos interdisciplinares relacionados ao tema foi realizada uma pesquisa com o objetivo de compreender a evolução, a interdisciplinaridade e as variáveis teórico-empíricas relacionadas à integração de tecnologias na educação básica brasileira. Este artigo apresenta os resultados da pesquisa exploratória realizada com procedimentos de revisão sistemática da literatura em bases de dados eletrônicas seguida de análises bibliométrica e descritiva. Entre os resultados obtidos, foram identificadas como

\footnotetext{
${ }^{1}$ Mestranda do Programa de Pós-Graduação em Tecnologias da Informação e Comunicação (PPGTIC) pela Universidade Federal de Santa Catarina. Bacharel em Tecnologias da Informação e Comunicação pela Universidade Federal de Santa Catarina (2013). Pesquisadora no Laboratório de Experimentação Remota (RExLab). Email: priscilanicolete@ hotmail.com

${ }^{2}$ Possui graduação em Engenharia de Produção Mecânica pela Universidade Federal de Santa Catarina UFSC (1997), mestrado (1999) e doutorado (2007) em Engenharia de Produção pela UFSC. Atualmente é professora com dedicação exclusiva da UFSC - Campus Araranguá. Email: simone.bilessimo@ufsc.br

3 Professora do Departamento de Engenharia do Conhecimento da Universidade Federal de Santa Catarina. Doutora em Engenharia e Gestão do Conhecimento. Email: patricia.sa.freire@ufsc.br

${ }^{4}$ Possui graduação em Administração de Empresas pela PUC-RS (1991), mestrado em Ciências da Computação pela Universidade Federal de Santa Caarina - UFSC (2002) e doutorado em Engenharia e Gestão do Conhecimento (2007). Professor adjunto da UFSC.Email: juarez.bento@ufsc.br

${ }^{5}$ Doutoranda em Engenharia e Gestão do Conhecimento pela Universidade Federal de Santa Catarina UFSC, Mestre em Ciência da Computação pela UFSC (2003). Especialista em Educação Inclusiva (URBRJ). Bacharel em Ciência da Computação pela UNISUL (2001). Pesquisadora do RexLab - Laboratório de experimentação Remota (UFSC). Email: marta.php@gmail.com
} 
estratégias para a integração de tecnologias na educação básica pública brasileira; Experimentação Remota para ensino de Física, Mobile Learning e Softwares para práticas nas aulas de Matemática. $\mathrm{O}$ estudo demonstrou a interdisciplinaridade do tema identificando seis grandes áreas do conhecimento: Matemática, Ciências Sociais, Ciência da Decisão, Ciência da Computação, Engenharias e Negócios/Administração.

PALAVRAS-CHAVE: Interdisciplinaridade. Tecnologia da Informação e da Comunicação. Educação. Ensino básico. Integração.

RESUMEN: La complejidad inherente al contexto de integración tecnológica en la educación básica - en la realidad actual de los estudiantes y en la cultura digital que domina la sociedad actual - caracteriza una transformación en la dinámica de la construcción del conocimiento, del cual los procesos de enseñanza y aprendizaje, utilizando métodos de enseñanza tradicional, presentan dificultades para conectarse al alumno e involucrarlo de forma plena en ese proceso. Ese nuevo contexto pasa a exigir investigaciones interdisciplinares para su mejor comprensión, involucrando especialistas en el área de ciencias sociales y áreas más técnicas como ingenierías y ciencia de la computación. Para responder la cuestión de cómo se procesa la evolución de los estudios teóricos y empíricos interdisciplinares relacionados al tema fue realizada una investigación con el objetivo de comprender la evolución, la interdisciplinaridad y las variables teórico-empíricas relacionadas a la integración de tecnologías en la educación básica brasileña. Este artículo presenta los resultados de la investigación exploratoria realizada con procedimientos de revisión sistemática de la literatura en bases de datos electrónicas seguida de análisis bibliométrico y descriptivo. Entre los resultados obtenidos, fueron identificadas como estrategias para la integración de tecnologías en la educación básica pública brasileña; Experimentación Remota para enseñanza de Física, Mobile Learning y software para prácticas en las clases de Matemáticas. El estudio aun demostró la interdisciplinaridad del tema, identificando 6 (seis) grandes áreas del conocimiento; Ciencias Sociales, Ciencia de la Decisión, Ciencia de la Computación, Ingenierías, Negocios/Administración y Matemáticas, demostrando la interdisciplinaridad del tema. PALABRAS CLAVE: Interdisciplinaridad. Tecnología de la Información y de la Comunicación. Educación. Enseñanza básica. Integración.

ABSTRACT: The complexity inherent to technological integration in the context of basic education in the current reality of the students and the digital culture that permeates society today features a transformation in the dynamics of knowledge construction. It is difficult to connect students, fully involving them in this process when using traditional teaching and learning methods. This new context requires interdisciplinary research for better understanding, involving experts in the field of sciences and more technical areas such as engineering and computer science. To answer the question of how to handle the evolution of interdisciplinary theoretical and empirical studies related to the subject, it was developed a survey in order to understand the evolution, interdisciplinarity and the theoretical and empirical variables related to the integration of technologies in the Brazilian basic education. This article presents the results of the exploratory survey of systematic review procedures of literature in electronic databases, then bibliometric and descriptive analyzes. Among the obtained results, were identified as strategies for the integration of technologies in Brazilian public basic education: Remote Experimentation for Physics, Mobile 
Learning and Software teaching for practices in Mathematics classes. The studies are divided into six large areas of knowledge: Social Sciences, Decision Science, Computer Science, Engineering, Business and Mathematics, demonstrating the interdisciplinarity of the theme.

KEYWORDS: Interdisciplinarity. Information and Communication Technologies. Education. Basic Education. Integration.

\section{Introdução}

Os estudos sobre a interdisciplinaridade surgiram a partir da necessidade de verificar de que forma o desenvolvimento de uma disciplina poderia dar contribuições ao desenvolvimento de outras, onde determinados problemas complexos não poderiam ser solucionados com métodos oriundos de uma única disciplina (BOSCO, 2012).

Com níveis de complexidade crescente, que surgem no mundo atual, muitas vezes decorrentes do próprio avanço dos conhecimentos científicos e tecnológicos, necessita-se da reformulação de currículos para resolver problemas específicos comuns de diferentes áreas (CAPES, 2011).

Na educação, apesar dos moldes tradicionais que as Instituições de Ensino (IE) ainda apresentam, discussões sobre a interdisciplinaridade vêm sendo travadas ao longo dos anos. Paulo Freire em 1987 já trazia esse conceito em seu livro "A pedagogia do Oprimido"; para ele a educação se fará tão mais pedagógica e tão mais crítica quando se quebrar os esquemas estreitos das visões "focalizadas" da realidade, aproveitando a compreensão da totalidade. Para Freire (1987), a interdisciplinaridade no ensino é o processo metodológico de edificação do conhecimento pelo sujeito com base na relação com o contexto, com a realidade e com sua cultura.

Nesse sentido, levando-se em conta a realidade atual dos estudantes, do qual vivem na chamada Cybercultura, compreende-se que houve uma transformação na dinâmica da construção do conhecimento (LEVY, 2010). Nas escolas, encontra-se uma nova geração de alunos, que vivem imersos em uma sociedade tecnológica, na qual instrumentos como computadores e dispositivos móveis fazem parte da cultura atual e da realidade do cotidiano. Os processos de ensino e aprendizagem se tornaram um tanto mais complexos, sendo que os métodos do ensino tradicional, comparativamente, apresentam dificuldades para prender a atenção dos alunos e fixar a aprendizagem.

Nessa nova realidade, se faz necessário o encontro de novas metodologias interdisciplinares de ensino, envolvendo o contexto e a realidade do aluno. É exigido da 
escola uma maior proximidade com o seu tempo e, para atender a esta demanda, o caminho hoje percorrido é a interconexão dos processos de ensino e aprendizagem com as Tecnologias de Informação e Comunicação (TIC), com o objetivo de aproveitar ao máximo o potencial de cada aluno, aperfeiçoando, desta forma, o ato de ensinar e aprender.

Ressalta-se a importância da integração e não da substituição de uma disciplina pela outra. A Unesco, no seu estudo sobre o uso dos aparelhos móveis na educação, aponta que projetos educacionais que se utilizem das TIC não são substitutos, e sim complementos de investimentos educacionais já existentes para a educação de qualidade, como infraestruturas, treinamento, livros e conteúdos (UNESCO, 2014).

Porém, mesmo reconhecendo a importância da integração tecnológica na educação, poucos são os estudos que analisam esta integração na educação básica.

Nesse contexto, elabora-se a questão de pesquisa: como se processa a evolução dos estudos teóricos e empíricos interdisciplinares relacionados às estratégias de integração tecnológica na educação básica pública brasileira e quais as variáveis propostas? Para responder a essa questão, definiu-se como objetivo desse estudo compreender a evolução e a interdisciplinaridade relacionadas às estratégias de integração tecnológica na educação básica pública brasileira, identificando as possíveis lacunas e oportunidades de futuras pesquisas sobre o tema.

Para o alcance deste objetivo o artigo está assim estruturado. As duas próximas seções apresentam as definições de "Educação Básica Pública" e "Integração de Tecnologias na Educação" por diferentes autores. Logo após, os procedimentos metodológicos são apresentados. De modo a auxiliar na compreensão do objeto de estudo, este artigo apresenta os resultados de uma revisão sistemática da literatura em bases de dados eletrônicas sobre as variáveis associadas, identificadas em estudos teóricos e empíricos.

Por fim, a análise da interdisciplinaridade do tema é mapeada e o artigo discorre sobre implicações teóricas e práticas desses resultados e propõe trabalhos futuros.

\section{Educação Básica Pública}

Os alicerces da qualidade no ensino superior são construídos desde a Educação Básica $^{6}$, ou seja, a qualidade da Educação Infantil, do Ensino Fundamental e do Ensino

${ }^{6}$ Sistema de ensino brasileiro é dividido em ensino básico e ensino superior. O ensino básico corresponde ao K12 (Kindergarten-12th grade) do sistema de ensino norte americano, contemplando crianças e jovens 
Médio é diretamente proporcional à qualidade da educação superior, tanto em termos de conteúdo quanto de ética e de responsabilidade.

O baixo número de graduados no Brasil está relacionado, entre outros fatores, com a falta de infraestrutura e matrículas no ensino médio, que pode ser apontado como o gargalo do sistema educacional brasileiro. Dados do IBGE (Instituto Brasileiro de Geografia e Estatística) indicavam que em 2013, 51,8\% dos jovens entre 15 e 17 anos estavam matriculados nessa etapa da educação básica. Isso significa que 48,2\% das pessoas desta a faixa etária ainda cursam o ensino fundamental ou já abandonaram seus estudos (IBGE, 2013).

Outro grande problema na educação brasileira é a elitização do ensino superior, apesar do número pequeno de alunos cursando o ensino básico na rede privada, de apenas $16,46 \%$, esses representam a maioria nas universidades públicas (INEP/MEC, 2013b). Em 2015, por exemplo, os aprovados para ingressarem na Universidade Federal de Santa Catarina (UFSC), vindos das escolas privadas, representaram 56,54\% enquanto a representação dos estudantes do ensino público é apenas de 43,46\% (COPERVE, 2015).

Esta desigualdade no acesso ao ensino superior relacionada aos jovens oriundos da escola pública deve-se, em parte, a pouca atenção dada à qualidade do Ensino Básico, a falta de investimentos na educação, as desigualdades educacionais entre as classes sociais e a seletividade nas formas de ingresso que dificultam o acesso a este nível de ensino, trazendo grandes desafios para os dias atuais.

Uma das consequências desta cisão entre Ensino Superior e Educação Básica é a reprovação continuada e o alto índice de evasão nos cursos de graduação. Apesar do crescimento dos últimos anos do número de matriculas no ensino superior, os números de concluintes continuam baixos. Como demonstrado na Tabela 1, conforme o Censo da Educação Superior (2013a), o número de concluintes em relação aos ingressantes não passa de $50 \%$ em todas as áreas.

dos 4 (quatro) aos 17 (dezessete) anos de idade. O ensino básico compreende três níveis; Ensino Infantil (pré-escolar), Ensino Fundamental (K1 à $\mathrm{K} 9)$ e Ensino Médio (K10 à K12). http://www.planalto.gov.br/ccivil_03/leis/19394.htm 
Tabela 1: Número de Matriculas, Ingressantes e Concluintes para cada 10.000 habitantes no Ensino Superior por área em 2013

\begin{tabular}{lrrr}
\hline Área Geral do Curso & Matrículas & Ingressantes & Concluintes \\
\hline Ciências sociais, negócios e direito & 147,20 & 56,40 & 21,80 \\
\hline Educação & 68,20 & 23,30 & 10,00 \\
Saúde e bem-estar social & 49,00 & 17,00 & 7,00 \\
Engenharia, produção e construção & 50,60 & 20,00 & 4,00 \\
Ciências, matemática e computação & 22,00 & 8,80 & 2,70 \\
Agricultura e veterinária & 8,90 & 2,80 & 1,00 \\
Humanidades e artes & 8,10 & 3,20 & 1,40 \\
Serviços & 8,30 & 4,20 & 1,40 \\
\hline
\end{tabular}

Fonte: autoras

Ao observar os números das áreas de exatas, "Engenharia, produção e construção" e "Ciências, matemática e computação", percebe-se que a situação fica ainda pior. Nas engenharias apenas $20 \%$ dos estudantes chegam ao final, e em "Ciências, matemática e computação", um pouco mais de $30 \%$ dos alunos consegue concluir seus estudos.

Diante do atual cenário, apresenta-se como imprescindível investir em novas metodologias de ensino e de aprendizagem na Educação Básica na rede pública. Considerando o novo perfil de estudantes que estão nas escolas básicas e ingressando na educação superior, autores discutem sobre a integração das TIC como possibilidade de implementação de conteúdos digitais para inovação das metodologias de ensino.

Desse modo, tenta-se transformar a educação tradicional em uma educação centrada no aluno de forma dinâmica e interativa, condizentes com a nova realidade cultural. Como afirma Da Silva (2011, p. 539), “as novas tecnologias estão influenciando o comportamento da sociedade contemporânea e transformando o mundo em que vivemos", de forma que "as tecnologias da informação, junto com a habilidade para usá-las e adaptá-las, são o fator crítico para gerar e possibilitar acesso à riqueza, poder e conhecimento no nosso tempo"(CASTELLS, 1999).

Neste contexto, é imprescindível que a Educação Básica seja repensada, em termos de país. Antes de pensarmos nos problemas de aprendizagem do ensino superior, é preciso olhar para a raiz do problema, que começa desde o ensino fundamental. É preciso combater a alta evasão no ensino fundamental, evitando a retenção de estudantes na transação para o ensino médio. E isso passa pela valorização e requalificação dos docentes, por questões curriculares e por melhor infraestrutura. Em relação às questões curriculares é necessário desenvolver e aplicar metodologias de ensino e de aprendizagem que se apropriem dos recursos disponibilizados pelas TIC 
desde as series iniciais, a fim de explorá-las como recursos auxiliares no apoio a construção de conceitos e na motivação desses estudantes (TAJRA, 2011).

\section{Integração de tecnologia na educação}

Tendo em vista a importância da utilização das TIC na educação, o governo brasileiro tem estimulado a inserção das Tecnologias de Informação e Comunicação através da criação de programas de incentivo. Entre estes programas, no Brasil, encontra-se o Programa Nacional de Tecnologia Educacional (ProInfo), desenvolvido pelo Ministério da Educação (MEC), que tem como objetivo promover o uso pedagógico da informática na rede pública de educação básica. O projeto visa fornecer às escolas computadores, recursos digitais e conteúdos educacionais.

Outro programa desenvolvido pelo MEC é o ProInfo Integrado. Funcionando como uma extensão do ProInfo, o projeto tem como objetivo promover a formação continuada aos profissionais para o uso didático-pedagógico das TIC no cotidiano escolar. Além disso, o Ministério da Educação desenvolve a cada dois anos um Guia de Tecnologias Educacionais, que pretende direcionar os profissionais ao conhecimento e ao uso das tecnologias disponíveis para a educação (MINISTÉRIO DA EDUCAÇÃO, 2013).

Apesar da conscientização do governo, a integração das tecnologias nas salas de aulas das escolas públicas ainda ocorre de forma lenta. Conforme Da Silva (2011), programas como Proinfo e Proinfo Integrado não têm obtido sucesso em suas implementações. A falta de infraestrutura, a desvalorização e a falta de qualificação dos professores continuam sendo fortes barreiras para desenvolver projetos inovadores nas escolas (DA SILVA, 2011).

Dados do Censo Escolar Brasileiro 2013 demonstram em números a carência de infraestrutura tecnológica sinalizada pela por Da Silva (2011). Apenas 44\% das escolas públicas possuem laboratórios de informática, e esses laboratórios contam, em médio, com apenas 8 computadores para uso dos alunos. Em relação à conectividade somente 50,8\% possuem conexão com a Internet (INEP/MEC, 2013b).

Nesse cenário, a Unesco (2014) alerta para o uso dos dispositivos móveis como aliado para a qualidade da educação. Conforme o relatório "Diretrizes de políticas para a aprendizagem móvel", afirma que atualmente as tecnologias móveis são muito mais 
comuns, mesmo em áreas onde escolas, livros e computadores são raros (UNESCO, 2014).

O desenvolvimento de tecnologias móveis e as suas aplicações e implicações no campo educacional surgiu recentemente como uma nova área de investigação, que traz uma nova forma de uso das TIC, a fim de acessar as informações através de dispositivos móveis, tais como os telefones, tablets, PDAs, MP3 players, drives flash USB, dispositivos de leitura eletrônicos e smartphones. Esta abordagem é comumente conhecida como Mobile Learning (m-learning) ou aprendizagem móvel. (SILVA et al., 2014).

Com a alta popularização, devido ao seu baixo custo, os dispositivos móveis são citados por muitos relatórios como uma alternativa viável para a utilização nas salas de aula. Com isso, o conceito Bring Your Own Technology (BYOD), traduzido para o português como Traga Seu Próprio Dispositivo, atualmente está sendo muito discutido ao redor do mundo como possibilidade para a educação. $O$ termo que inicialmente foi criado pela Intel com o intuito de melhorar a produtividade de seus funcionários, hoje já é uma alternativa também para as escolas (JOHNSON et al., 2014).

Atualmente, o significado que os dispositivos móveis têm na vida das pessoas, em especial para os jovens, permite tirar proveito do que sua onipresença tem para oferecer (DE LIMA et al., 2014). Os estudantes estão entrando na sala de aula com os seus próprios dispositivos, o que representa uma oportunidade para as escolas públicas para desenvolver metodologias inovadoras com o uso das Tecnologias de Informação e Comunicação. "BYOD traz implicações profundas para a educação primária e secundária, pois cria condições para a ocorrência da aprendizagem centrada no aluno" (JOHNSON et al., 2014, p 35).

\section{Procedimentos Metodológicos}

Esta pesquisa pode ser classificada como exploratória utilizando-se de procedimentos de revisão sistemática da literatura em bases de dados eletrônicas, propondo-se a realizar análises bibliométrica e descritiva. 


\section{Revisão Sistemática da Literatura}

Para determinar a confiabilidade da pesquisa quanto aos seus resultados e análises, bem como ser possível replicar seus procedimentos, foi utilizada a revisão sistemática da literatura e as análises bibliométrica e descritiva, conforme orientação de Freire (2013).

A Revisão Sistemática da Literatura (RSL) é um método de pesquisa exploratória que permite explicitar todos os passos realizados para o desenvolvimento de uma pesquisa bibliográfica. Conforme Sampaio e Mancini (2007), a RSL é uma forma de pesquisa que utiliza como fonte de dados a literatura para reunir estudos semelhantes a fim de identificar, selecionar e avaliar criticamente um tema. É "a aplicação das estratégias científicas que limitam o viés de seleção e avaliam com espírito científico os artigos e sintetizam todos os estudos relevantes em tópicos específicos" (PERISSÉ; GOMES; NOGUEIRA, 2001)

A RSL permite através da análise exploratória aclarar as variáveis trabalhadas de um tema e identificar variáveis do estudo mais atuais, permitindo a identificação de novas tendências e oportunidades de novos estudos.

Para o desenvolvimento de uma RSL eficiente é necessária a utilização de algumas técnicas para definir passos a serem seguidos. Conforme Dagostin, Freire e Guimarães Filho (2014), podem ser utilizados dois tipos de orientações para a elaboração de uma RSL. São eles: “Cochrane Handbook 1 (Colaboração Cochrane) e; CDR Report6 4 (NHS Centre for Reviews and Dissemination, University of York)”.

\footnotetext{
O Cochrane Handbook determina a sequência de sete passos para a RSL, são eles: Formulação da pergunta; Localização e seleção das bases de dados para pesquisa; Avaliação crítica dos estudos; Coleta de dados; Análise e apresentação dos dados; Interpretação dos dados; Aprimoramento e atualização da revisão. O Manual NHS/York2 determina nove fases distribuídas em três estágios: Planejamento, Execução e Apresentação (DAGOSTIN; FREIRE; GUIMARÃES FILHO, 2014, p.4).
}

Ambos os manuais foram desenvolvidos para pesquisas na área da saúde, com objetivo de esclarecer um quadro clínico através de revisão sistemática baseada em evidências, e dessa forma ajudar os profissionais de saúde a diagnosticar e sugerir tratamentos eficientes para determinadas doenças.

Cochrane é uma rede global independente de profissionais de saúde e pesquisadores que possuem o desafio de tornar a grande quantidade de evidência gerada através de pesquisas úteis para informar decisões sobre a saúde. Cochrane Handbook "é 
reconhecido internacionalmente como o ponto de referência para informações de qualidade sobre a eficácia dos cuidados de saúde" (COCHRANE, 2015). A revisão Cochrane é uma investigação científica, com uma sequência de métodos pré-planejados e um conjunto de estudos originais. Cada revisão sistemática aborda uma questão claramente formulada. Os resultados destas investigações primárias são sintetizados por meio de estratégias que limitam viés e erro aleatório (COCHRANE, 2015).

Da mesma forma, o CDR Report6 da NHS/York2 tem como objetivo promover elevados padrões de pesquisa, fornecendo orientações práticas para a realização de revisões sistemáticas que avaliam os efeitos de intervenções de saúde (CRD, 2009). O Manual da NHS/York2 possui reconhecimento mundial, e produz política de investigação relevante e métodos inovadores que promovam o uso de evidências de pesquisa para melhorar a saúde da população (CRD, 2009).

Ambas as metodologias possuem estratégias bem definidas que incluem uma pesquisa abrangente de todos os estudos potencialmente relevantes e a utilização de critérios explícitos e reproduzíveis de seleção de estudos para avaliação. Por esse motivo, atualmente, essas metodologias são utilizadas amplamente para diversas áreas do conhecimento. Na pesquisa aqui apresentada, o método utilizado para seleção e avaliação foi o Cochrane Handbook, com as suas sete fases de desenvolvimento (Figura $1)$.

Figura 1: Fases do Cochrane Handbook

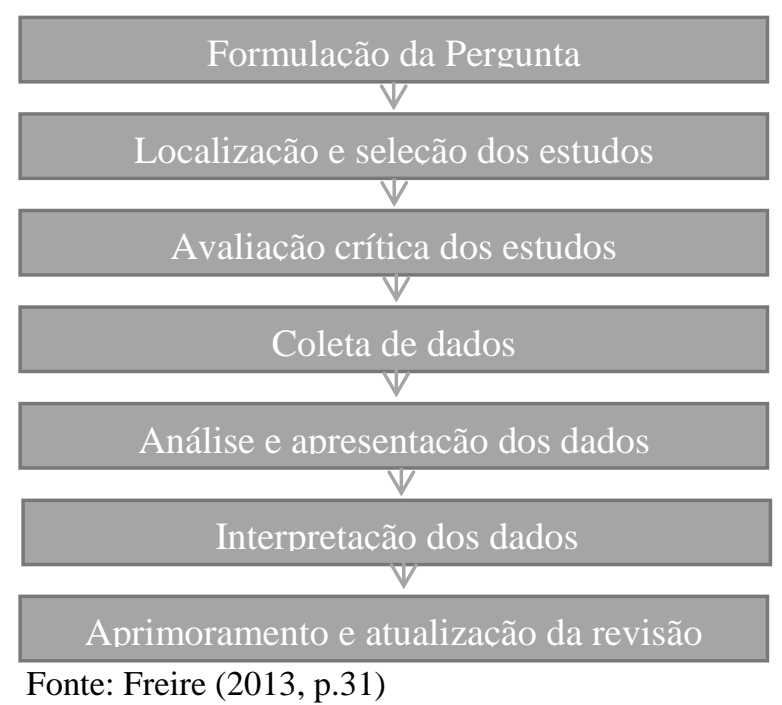

Para a análise dos resultados foram utilizadas as técnicas de análise descritiva e bibliométrica. 
Conforme Dagostin, Freire e Guimarães Filho (2014) e Freire (2013), a análise descritiva é um tipo de análise qualitativa que é utilizada para desenvolver um agrupamento dos dados analisados. Gil (2008, p. 28) acrescenta que "as pesquisas deste tipo têm como objetivo primordial a descrição das características de determinada população ou fenômeno ou o estabelecimento de relações entre variáveis".

A bibliometria é uma técnica de investigação para avaliar resultados de uma pesquisa bibliográfica sobre uma determinada questão de pesquisa. Tem por objetivo a análise do tamanho, crescimento e distribuição da bibliografia num determinado campo do conhecimento, permitindo assim mapear e gerar indicadores de tratamento e gestão da informação e do conhecimento (FREIRE, 2013). Essa técnica permite indicar tendências, analisar o impacto da produção científica de um determinado tema, estudar indicadores e mapear frequência e lugar onde o tema é mais explorado (FREIRE, 2013).

\section{Planejamento}

Seguindo o manual Cochrane Handbook foram realizados alguns passos para a cumprimento desse estudo. Primeiramente, foi definido como pergunta da revisão: “como se processa a evolução dos estudos teóricos e empíricos interdisciplinares relacionados às estratégias de integração tecnológica na educação básica pública brasileira e quais as variáveis propostas?", identificado como variáveis:

Variável 1: Estratégias de integração tecnológica

Variável 2: Educação básica pública brasileira

\section{Unidade de Observação: Brasil}

Optou-se pelo banco de dados SCOPUS, por ele possuir mais de 16.500 títulos de mais de 4.000 editores internacionais; 575 publicações comerciais; 315 séries de livros; 3,6 milhões de papers de conferências e 37 milhões de registros que permitem explorar o tema em um nível macro de estudos.

Para fins de pesquisa na base de dados, foram selecionadas as seguintes palavras-chaves, de forma hierárquica: “Basic Education”, Technolog* e Integration, ao final aplicando um filtro geográfico: Brasil. 


\section{Estratégias da Execução}

$\mathrm{Na}$ base de dados Scopus foi primeiramente utilizado o termo "basic educat*". A busca realizada com tal palavra-chave ocorreu em: títulos, resumo (abstract), palavras-chave (keywords). Desta forma, foram encontradas 2.727 (dois mil setecentos e vinte e sete) publicações. Após esse resultado, foi refinada a busca utilizando a palavra-chave "technolog*", retornando 589 (quinhentos e oitenta e nove) publicações. O filtro seguinte foi a palavra-chave "Integrat*", assim obtendo 153 (cento e cinquenta e três) publicações.

Por ser uma pesquisa centrada nos estudos realizados no Brasil, na sequência da aplicação dos filtros no sistema de busca, incluiu-se a palavra Brasil como opção. Com a utilização do filtro resultaram 17 (dezessete) publicações.

Nesta etapa, realizou-se uma leitura do título, resumo e introdução das 17 (dezessete) publicações encontradas. Destes, 5 (cinco) artigos foram excluídos por não tratarem do tema proposto neste documento, são eles:

- "A Relação entre a educação profissional e a educação básica na CONAE 2010: possibilidades e limites para a construção do novo Plano Nacional de Educação" de Moura (2010).

- "Education for Science and Science for Education: More than a Play upon Words", de Grynszpan e Araújo-Jorge (2000).

- "O desempenho acadêmico como indicador de qualidade da transição Ensino Médio-Educação Superior", de Fagundes, Luce e Espinar (2014).

- "Permanência e evasão na educação técnica de nível médio em Minas Gerais", de Dore e Lüscher (2011).

- "Trabalho docente na Educação Profissional e Tecnológica e no PROEJA", de Shiroma e Lima Filho (2011).

Ao final da aplicação dos filtros e dos critérios de exclusão foram selecionados para a análise (12) doze artigos, que configuram-se na amostra final.

\section{Apresentação e Análise dos Resultados}

Para responder à questão de pesquisa, de como se processa a evolução dos estudos teóricos e empíricos interdisciplinares relacionados às estratégias de integração tecnológica na educação básica pública brasileira e quais as variáveis propostas, foi necessário construir a linha do tempo das publicações coletadas e realizar um mergulho em seus resumos e artigos completos para identificar as variáveis trabalhadas por cada um deles. Assim, uma análise bibliométrica e descritiva tiveram que ser realizadas. 


\section{Análise Bibliométrica}

Dando início à análise bibliométrica sobre a linha do tempo das publicações, pode-se perceber uma maior incidência de publicações nos anos de 2011 e 2014, possuindo quatro publicações em cada ano. Nos anos entre essas duas datas houve uma redução nas publicações, sendo que 2012 e 2013 apresentaram apenas 2 publicações cada (Gráfico 1).

Gráfico 1: Publicações por ano

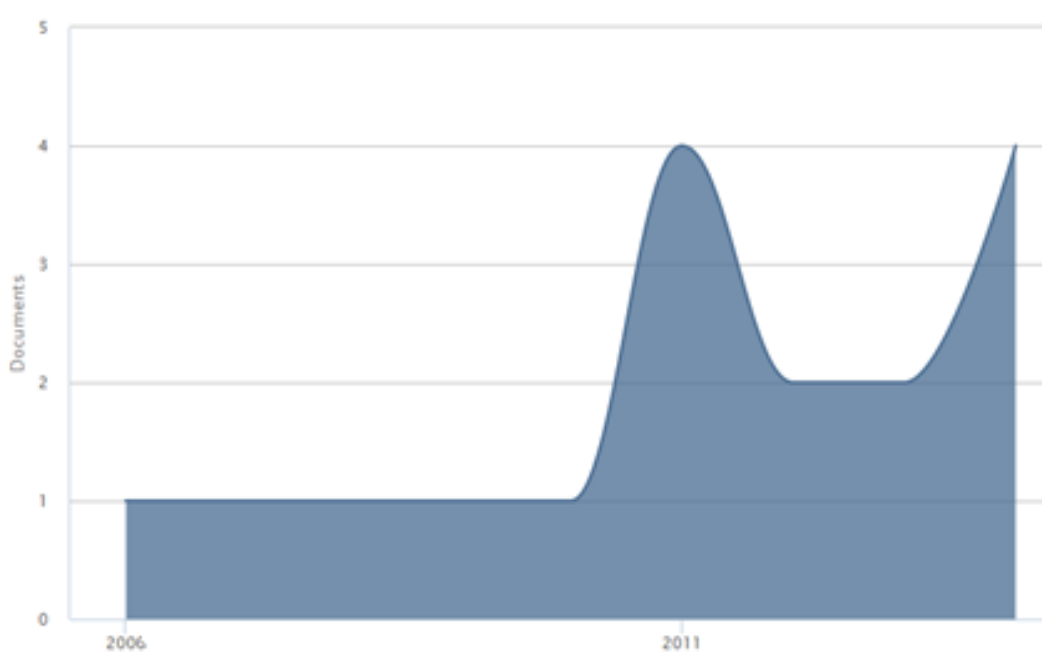

Fonte: Dados da pesquisa (Scopus, 2015)

O primeiro artigo identificado sobre o tema é de 2006, dos autores Franco, Cruz e Lopes. O documento traz interdisciplinaridade entre as áreas da Geografia, Geometria, Línguas e Artes, através da utilização de gráficos de computador, tecnologias interativas e aprendizagem colaborativa. Os artigos mais recentes, de 2014, trazem a atual tendência da Mobile Learning, com a crescente popularidade dos dispositivos móveis e a Experimentação Remota como alternativa para o ensino das áreas STEM (Ciência, Tecnologias, Engenharia e Matemática), considerando o baixo número de laboratórios de ciências nas escolas básicas da rede pública.

Referente ao tipo de publicações, as publicações se dividiram em Artigos apresentados em conferências e artigos publicados em periódicos, com $42 \%$ e $50 \%$ respectivamente. Tendo apenas um capítulo de livro publicado sobre o tema (Gráfico 2). 
Gráfico 2: Tipos de publicações

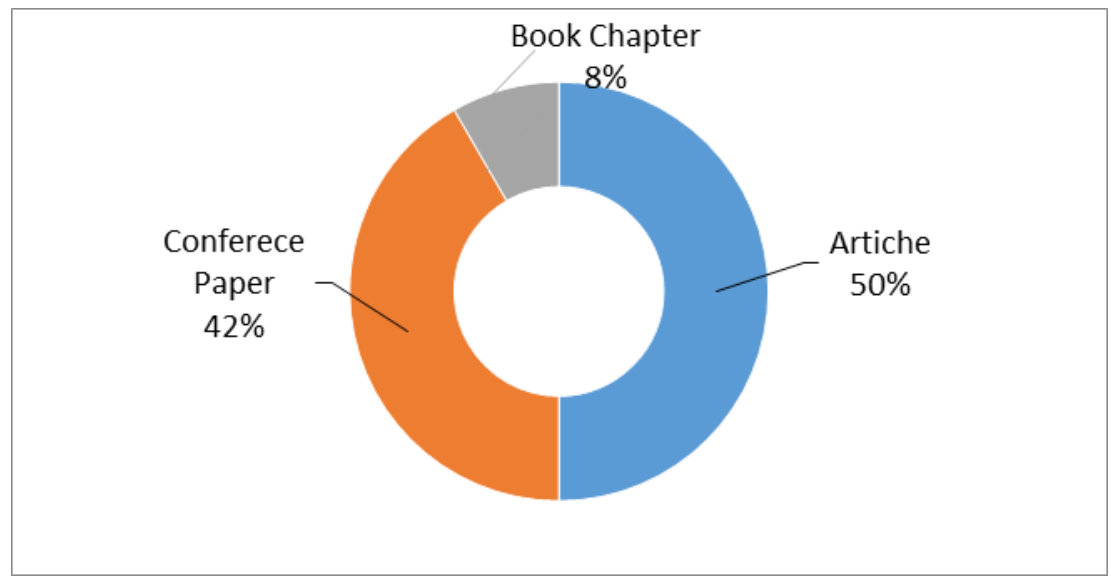

Fonte: dados da pesquisa (Scopus, 2015)

Ao realizar uma análise mais aprofundada das publicações utilizadas no presente estudo, verifica-se que a revista brasileira Educação \& Sociedade foi a que mais publicou sobre o tema (Gráfico 3). Entre as publicações, três artigos foram em periódicos internacionais: Revista Iberoamericana de Tecnologias del Aprendizaje, Proceedings Frontiers in Education Conference e Espacios.

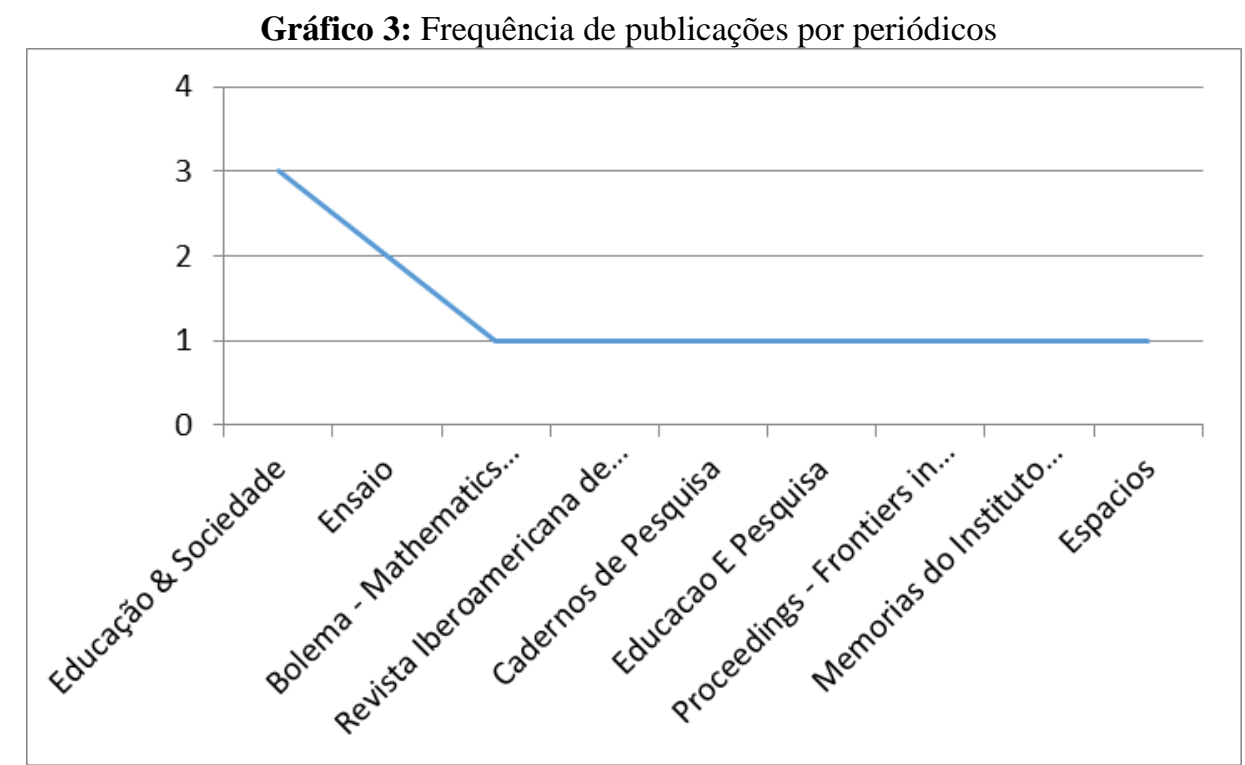

Fonte: dados da pesquisa (Scopus, 2015)

Entre as afiliações, a Universidade Federal de Santa Catarina (UFSC) é a que mais tem dado atenção ao tema, com 4 (quatro) publicações. Na sequência, com 2 (duas) publicações cada, a Universidade Estadual de Campinas, Universidade de São Paulo, Universidade Federal do Rio Grande do Sul e o Instituto Superior do Porto, que publicou artigos em parceira com a UFSC (Gráfico 4). 
Gráfico 4: Afiliações dos autores que publicaram sobre o tema

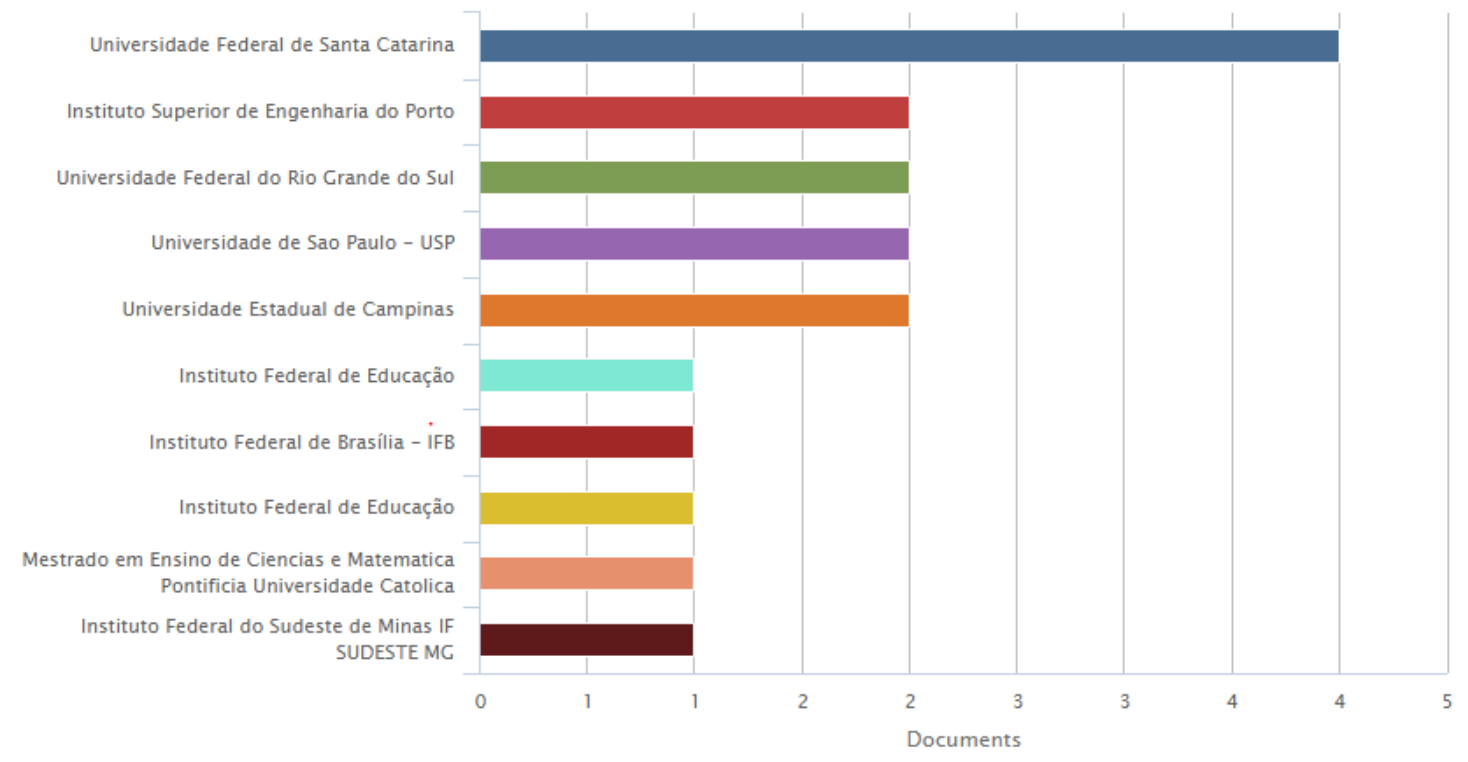

Fonte: dados da pesquisa (Scopus, 2015)

O Gráfico 5 apresenta os autores que mais contribuíram em publicações. Shardosim (2013, 2014, 2014), Silva (2013, 2014, 2014) e Rochadel (2013, 2014, 2014), possuem 3 artigos cada.

Gráfico 5: Publicações por autor

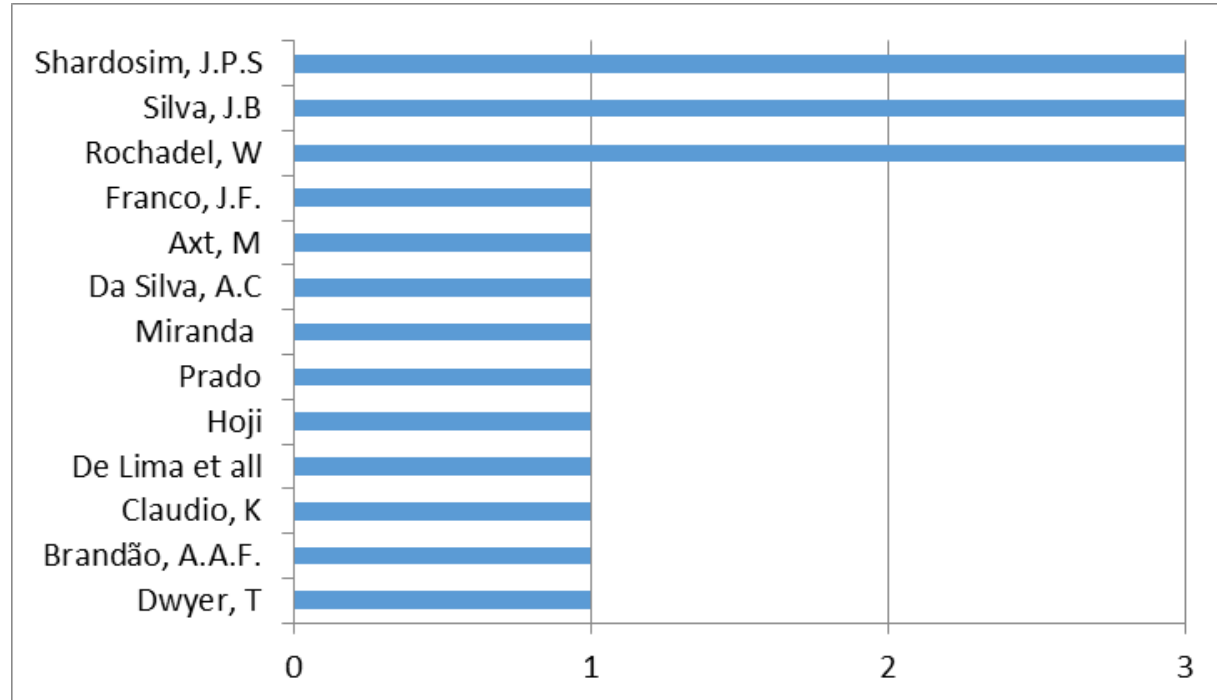

Fonte: dados da pesquisa (Scopus, 2015)

Estre os artigos analisados, dois possuem citações registradas na Scopus. O artigo "Desvendando mitos: Os computadores e o desempenho no sistema escolar" do autor Dwyer et al (2007) alcançou três citações, e o artigo "Application of remote 
experiments in basic education through mobile devices" do autor De Lima et al (2014) aparece com uma citação.

\section{Análise Descritiva}

Uma das perguntas mais pertinentes quando se discute a integração de tecnologias na sala de aula é: As tecnologias nas salas de aula favorecem os processos de ensino $e$ aprendizagem? Pela análise descritiva realizada sobre os artigos coletados nesta pesquisa, pode-se afirmar que não há consenso para se concluir uma resposta.

Tabela 2: Detalhamento dos artigos analisados

\begin{tabular}{|c|c|c|c|c|}
\hline Autor & Ano & $\begin{array}{c}\text { Favorável ao } \\
\text { uso das TIC nas } \\
\text { Educação }\end{array}$ & Estratégia & Área de Ensino \\
\hline Dwyer et al & 2007 & Não favorável & Sem estratégia & $\begin{array}{l}\text { - Matemática } \\
\text { - Português }\end{array}$ \\
\hline $\begin{array}{l}\text { De Lima et } \\
\text { al }\end{array}$ & 2014 & Favorável & $\begin{array}{l}\text { - Experimentação Remota } \\
\text { - Mobile Learning } \\
\text { - AVA }\end{array}$ & • Física \\
\hline Silva et al & 2014 & Favorável & $\begin{array}{l}\text { - Experimentação Remota } \\
\text { - Mobile Learning } \\
\text { - AVA }\end{array}$ & - Física \\
\hline $\begin{array}{l}\text { Rochadel et } \\
\text { al }\end{array}$ & 2013 & Favorável & $\begin{array}{l}\text { - Experimentação Remota } \\
\text { - Mobile Learning } \\
\text { - AVA }\end{array}$ & - Física \\
\hline $\begin{array}{l}\text { Hoji, Vianna } \\
\text { e Félix }\end{array}$ & 2012 & Favorável & - Software Octave & - Matemática \\
\hline $\begin{array}{l}\text { Prado, } \\
\text { Brandão e } \\
\text { Brandão }\end{array}$ & 2011 & Favorável & $\begin{array}{l}\text { - Software iGraf } \\
\text { - LMS }\end{array}$ & - Matemática \\
\hline $\begin{array}{l}\text { Miranda e } \\
\text { Gazire }\end{array}$ & 2012 & Favorável & $\begin{array}{l}\text { - CD } \\
\text { - Vídeos } \\
\text { - Software Geogebra } \\
\text { - Software Winplot } \\
\end{array}$ & $\begin{array}{l}\text { - Interdisciplinar Saúde e } \\
\text { Matemática }\end{array}$ \\
\hline $\begin{array}{l}\text { Da Silva, Ã. } \\
\text { C }\end{array}$ & 2011 & Favorável & - PROINFO & - Geral \\
\hline Axt et al & 2008 & Favorável & $\begin{array}{l}\text { - Mega Vitta, jogo e } \\
\text { simulação de assistente } \\
\text { inteligente }\end{array}$ & - Educação Ambiental \\
\hline $\begin{array}{l}\text { Franco, } \\
\text { Cruz } \\
\text { Lopes }\end{array}$ & 2006 & Favorável & $\begin{array}{l}\text { - Gráficos de Computador } \\
\text { - Tecnologias Interativas } \\
\text { - Aprendizagem } \\
\text { Colaborativa }\end{array}$ & $\begin{array}{l}\text { - Interdisciplinar Geografia, } \\
\text { Geometria, Línguas e Artes. }\end{array}$ \\
\hline
\end{tabular}

Fonte: Autoria própria.

Dweyer et al (2007) tem uma opinião polêmica sobre o uso de tecnologias no ensino. Em seu artigo "Desvendando mitos: os computadores e o desempenho no sistema escolar", o autor defende que as TIC não favorecem nos processos de ensino e 
aprendizagem. O seu estudo, ao utilizar dados da pesquisa do SAEB para verificar o desempenho de alunos de $4^{\mathrm{a}}$ e $8^{\mathrm{a}}$ série do ensino fundamental e da $3^{\mathrm{a}}$ série do ensino médio, e a relação deste desempenho com o uso de computador, conclui que independente da série cursada e da classe social, o uso intenso do computador diminui o desempenho escolar (DWYER et al., 2007).

Ao analisar as conclusões da grande maioria dos autores (DE LIMA et al., 2014), (SILVA et al., 2014), (ROCHADEL et al., 2013), (HOJI; VIANNA; FELIX, 2012), (PRADO; BRANDÃO; BRANDÃO, 2011), (AXT et al., 2008), (FRANCO; DA CRUZ; LOPES, 2006), (STÊNICO; SILVA, 2014), (MOURA, 2013), pode-se perceber que há uma forte crença entre os autores que publicam sobre o tema no Brasil de que a tecnologia deva ser utilizada no ensino.

Onze entre os doze artigos analisados privilegiam a utilização das tecnologias em aula, mesmo fazendo ressalvas para seu uso, como o alerta de que a integração da tecnologia no ambiente escolar dependente do planejamento cuidadosamente realizado.

Da Silva (2011) defende que o uso das tecnologias deve ser planejado e feito de forma eficiente. A autora faz uma crítica ao programa do governo PROINFO, do qual afirma que não compreende a um programa eficaz de inclusão de tecnologias na educação. Para ela, além de equipamentos de qualidade e em quantidade, deve-se pensar na qualificação do docente que irá utilizar essas tecnologias na sala de aula. O autor ainda aponta a necessária valorização do docente a fim de motivá-lo a sair de sua zona de conforto do quadro e giz, e passar a utilizar novas formas de ensino.

Corroborando, Franco, Da Cruz e Lopes (2006) levantam a importância da intervenção pedagógica a fim de orientar e estimular o melhor uso das TIC nos processos de ensino e aprendizagem. Os autores relatam a utilização de gráficos de computador, tecnologias interativas e aprendizagem colaborativa para o ensino interdisciplinar nas disciplinas de geografia, geometria, línguas e artes.

Os trabalhos de De Lima et al (2014), Silva el al (2014) e Rochadel (2013) exploram, como estratégia de integração de tecnologias na educação, a utilização de Experimentação Remota (ER), Mobile Learning e Ambientes Virtuais de Aprendizagem (AVA) para o ensino de Física nas escolas de educação básica pública. Segundo De Lima et al (2014), "Experimentação Remota (ER) é uma tecnologia que permite ao usuário controlar um experimento real a partir de uma fonte remota, observando os resultados através de vídeo streaming”. Nesse sentido, para os autores, a utilização de ER e a Mobile Learning nas escolas públicas do país pode minimizar os problemas 
enfrentados pelas escolas, referentes à precariedade de infraestrutura dos laboratórios de ciências e laboratórios de informática.

Silva et al (2014) destacam a importância de aproveitar as tecnologias para melhorar o ensino das disciplinas que envolvam conceitos das áreas STEM (Ciência, Tecnologias, Engenharia e Matemática) para tornar essas disciplinas mais atraentes, a fim de motivar mais estudantes a seguirem nessas áreas, pois, segundo o autor, a diminuição do número de engenheiros e profissionais de TI preocupam diversos países ao redor do mundo. Disciplinas como Matemática, Física e Química muitas vezes são ensinadas simplesmente de forma teórica, o que leva os estudantes a não saberem o real sentido dos conceitos ensinados, levando por muitas vezes a desmotivação no ensino básica.

Nesse sentido, o trabalho de Hoji, Vianna e Felix (2012) e Prado, Brandão e Brandão (2011) também traz a importância de unir a teoria com a prática no ensino de matemática com a integração das Tecnologias da Informação e Comunicação. Os autores utilizam o Software Octave, que é uma ferramenta computacional numérica, e de conceitos matemáticos envolvidos, a fim de encontrar soluções de problemas aplicação.

Prado, Brandão e Brandão (2011) trazem uma proposta de integração do Software iGraf com um Sistema de Gestão de Aprendizagem, (LMS - Learning Management Systems). O Software iGraf, permite que os professores desenvolvam atividades que envolvam funções matemáticas (como desenhar gráficos, derivadas e integrais de funções), e os alunos podem interagir diretamente com atividades iGraf, através da Web. Conforme os autores, com a integração das duas ferramentas, o LMS proporciona um mecanismo de avaliação automática com feedback imediato aos alunos a partir das atividades realizadas no iGraf.

O artigo de Stênico e Silva (2014), "Educação profissional brasileira: Análises, discussões e tendências", não aborda diretamente a integração das tecnologias na escola, porém entre suas discussões sobre ligação entre educação básica e educação profissional, o autor sinaliza a tendência de criação de projetos nas escolas que contemplem as competências necessárias na atual sociedade, que envolve a ampla utilização das TIC no mercado de trabalho.

Artigo de Moura (2013), "Ensino médio integrado: subsunção aos interesses do capital ou travessia para a formação humana integral?”, também não explora estratégias de integração de tecnologias no ensino básico, mas trata a tecnologia como eixos 
estruturantes que fazem parte da formação humana integral, juntamente com o trabalho, a ciência e a cultura.

Assim, a partir da meta análise realizada, pode-se inferir que, mais do que $90 \%$ dos artigos, onze entre doze, afirmam que as tecnologias nas salas de aula favorecem os processos de ensino e aprendizagem.

\section{Análise da Interdisciplinaridade do Tema}

Ao analisar a interdisciplinaridade do tema Integração Tecnológica na Educação Básica Pública Brasileira, encontrou-se 6 (seis áreas) envolvidas. Conforme Gráfico 6 percebe-se que a maior concentração de publicações por área de estudo é em Ciências Sociais, com 75\%. As áreas de Ciência da Decisão, Ciência da Computação e Engenharias se dividem em $25 \%$ cada. As áreas de Negócios/Administração e Matemática ficam com a menor concentração de publicações, com 1\% cada.

Gráfico 6: Áreas de estudo

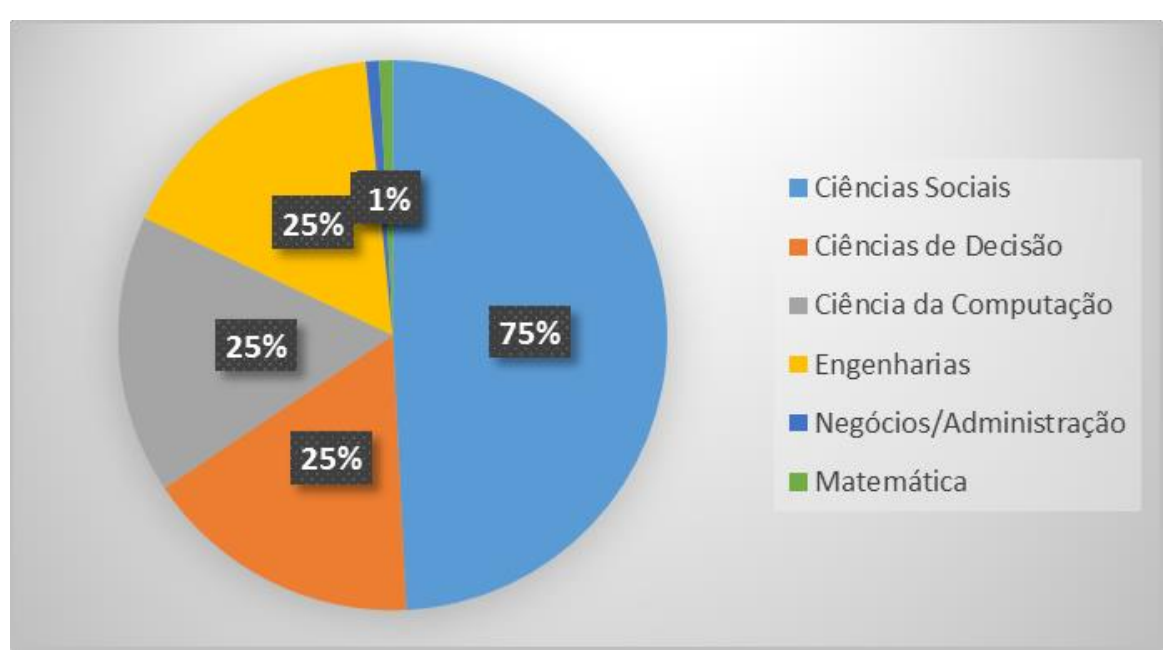

Fonte: dados da pesquisa (Scopus, 2015)

Ao verificar que existe uma maior concentração de publicações em uma das áreas, em um primeiro momento, pode-se constatar que o tema não compreende um estudo interdisciplinar, entretanto, ao analisar os artigos com maior profundidade percebe-se que a grande concentração na área de Ciência Sociais deve-se a estudos que contemplam mais de uma área de estudo.

Nesse sentido, muitas publicações demostram interdisciplinaridade em seu próprio trabalho, sendo enquadrado em mais de uma área de estudo. Os artigos do autores De Lima et al (2014) e Rochadel et al (2013) contemplam 3 áreas do 
conhecimento; Engenharia, Ciências Sociais e Ciências da Decisão. O trabalho de Silva et al (2014) se enquadra nas áreas de Engenharia e Ciências Sociais. Os artigos dos autores Hoji et al (2012) e Prado et al (2011), contemplam as áreas de Ciência da Computação e Ciências Sociais. Miranda e Gazire (2012) compreende as áreas de Matemática e Ciências Sociais e o artigo de Stênico e Silva (2014), Ciências da Decisão e Administração.

\section{Considerações Finais}

O estudo apresentado nesse documento objetivou compreender a evolução, a interdisciplinaridade e as variáveis teórico-empíricas relacionadas às estratégias de integração tecnológica na educação básica pública brasileira. Desse modo, pode-se perceber que a inserção de recursos tecnológicos na Educação Básica Pública requer mais do a simples integração da tecnologia no dia-a-dia de professores e alunos de forma indiscriminada. É preciso compreender o processo cíclico da educação em todos os seus níveis e com o conhecimento sobre cada nível. Compreender que as ações que preparam os adolescentes para seu ingresso na universidade estão diretamente ligadas à motivação impugnada desde o ensino fundamental, passando pelo ensino médio, estimulando o estudante a seguir na carreira acadêmica, escolhendo sua área de formação profissional de forma consciente e crítica.

A pesquisa bibliográfica aqui descrita evidencia que muitos trabalhos são desenvolvidos com a perspectiva de melhorar a educação por meio da integração tecnológica. É perceptível que a maioria das ações voltam-se para disciplinas essenciais em todos os níveis educacionais, como as disciplinas de Física e Matemática, por exemplo. Vale destacar que entre as estratégias mais utilizadas para essa integração, algumas envolvem a Experimentação Remota para ensino de Física, Mobile Learning e Softwares para práticas nas aulas de Matemática.

Ao verificar a interdisciplinaridade do tema, pode-se compreender que o tema "Integração de tecnologias na educação básica pública brasileira" é intrinsicamente interdisciplinar, apresentando estudos nas áreas de Ciências Sociais, Ciência da Decisão, Ciência da Computação, Engenharias, Negócios/Administração e Matemática. Além disso, muitas pesquisas compreendem mais de uma área do conhecimento, 
comprovando a necessidade do olhar interdisciplinar para compreensão deste tema como constructo científico.

Nesse sentido, diante da diversidade de aplicações em diferentes disciplinas, mas com abordagens em áreas de conhecimento distintas, torna-se evidente que a interdisciplinaridade é fundamental para que a tecnologia, enquanto mídia do conhecimento, seja utilizada como auxiliadora de processos de ensino e aprendizagem cada vez mais integradores e eficazes.

Para trabalhos futuros, sugere-se a realização da RSL com descritores relacionados aos sistemas americanos e europeus. Esta pesquisa teve foco no cenário brasileiro, e por esse motivo foram utilizados termos relacionados ao sistema brasileiro, como "Ensino Básico", “Ensino Fundamental” e "Ensino Médio”. Assim, sugere-se a realização de novas pesquisas relacionadas a outros sistemas de educação, empregando termos como: "Educação Primária”, "Educação Secundária” e “K-12”.

\section{REFERÊNCIAS}

AXT, M. et al. Maga Vitta: conversational ecological agent in an interactive collective construction environment for basic education. Intelligent Agents for Education, p. 73$95,2008$.

\section{BOSCO, J. Teoria Geral dos Sistemas: Em Busca da Interdiciplinaridade.}

Florianópolis: Instituto Stela, 2012.

CAPES - COORDENAÇÃO DE APERFEIÇOAMENTO DE PESSOAL DE NÍVEL SUPERIOR . Documento de área interdisciplinar: triênio 2007 - 2009. 2011

CASTELLS, M. A Sociedade em rede: A era da informação: economia, sociedade e cultura. São Paulo: Paz e Terra, 1999.

COCHRANE COMMUNITY. Cochrane Community (beta): Trusted evidence. Informed decisions. Better health., 2015.2 Disponível em: < http://community.cochrane.org/about-us >. Acesso em: 17 out. 2015.

COPERVE. Comissão Permanente do Vestibular. 2015.

CRD - CENTRE FOR REVIEWS AND DISSEMINATION. Systematic reviews: CRD's guidance for undertaking reviews in health care. Centre for Reviews and Dissemination, 2009. ISBN 1900640473.

DA SILVA, A. C. Educação e tecnologia: entre o discurso e a prática. Revista Ensaio: Avaliação e Políticas Públicas em Educação, v. 19, n. 72, p. 527-554, 2011.

DAGOSTIN, N. T.; FREIRE, P. D. S.; GUIMARAES FILHO, L. P. INOVAÇÃO PARA O DESENVOLVIMENTO SOCIOECONÔMICO: UM ESTUDO BIBLIOMÉTRICO. 2014. 
DE LIMA, J. et al. Application of remote experiments in basic education through mobile devices. Global Engineering Education Conference (EDUCON), 2014 IEEE, 2014. IEEE. p.1093-1096.

DORE, R. S.; LUSCHER, A. Z. Permanência e Evasão na Educação Técnica de nível médio em Minas Gerais. Cadernos de Pesquisa, v. 772, 2011.

DWYER, T. et al. Revealing myths: Computers and school performance. Educação \& Sociedade, v. 28, n. 101, p. 1303-1328, 2007.

MEC - MINISTÉRIO DA EDUCAÇÃO. Guia de Tecnologias. 2013. Disponível em: < http://portal.mec.gov.br/guia-de-tecnologias >. Acesso em: 17 out. 2015.

FAGUNDES, C. V.; LUCE, M. B.; ESPINAR, S. R. O desempenho acadêmico como indicador de qualidade da transição Ensino Médio-Educação Superior. Revista Ensaio: Avaliação e Políticas Públicas em Educação, v. 22, n. 84, p. 635-670, 2014.

FRANCO, J. F.; DA CRUZ, S. R.; LOPES, R. D. D. Computer graphics, interactive technologies and collaborative learning synergy supporting individuals' skills development. ACM SIGGRAPH 2006 Educators program, 2006. ACM. p.42.

FREIRE, P. Pedagogia do oprimido. Rio de Janeiro: Paz e Terra, 1987.

FREIRE, P. D. S. Aumente a qualidade e quantidade de suas publicações científicas. 1. Curitiba, PR: Editora CRV, 2013.

GIL, A. C. Métodos e técnicas de pesquisa social. São Paulo: Atlas, 2008.

GRYNSZPAN, D.; ARAÚJO-JORGE, T. C. Education for Science and Science for Education: more than a Play upon Words. Memórias do Instituto Oswaldo Cruz, v. 95, p. 49-52, 2000.

HOJI, E. S.; VIANNA, W. B.; FELIX, T. D. A computer-aided math teaching approach for students in a technical institute: The experience with the Octave in the electromechanical technical course. Interactive Collaborative Learning (ICL), 2012 15th International Conference on, 2012. IEEE. p.1-5.

IBGE. Instituto Brasileiro de Geografia e Estatística. 2013. Disponível em: < http://www.ibge.gov.br/ >. Acesso em: 17 out. 2015.

INEP/MEC. Censo da Educação Superior no Brasil 2013. 2013a

Censo Escolar Brasileiro 2013. Brasil, 2013b. Disponível em: < http://portal.inep.gov.br/basica-censo >. Acesso em: 17 out. 2015.

JOHNSON, L. et al. NMC Horizon Report: Edição K12. Austin, Texas, Estados Unidos. 2014

LEVY, P. Cibercultura. Editora 34, 2010. ISBN 8573261269.

MIRANDA, P. R. D.; GAZIRE, E. S. Saúde e Números: uma parceria de sucesso. Boletim de Educação Matemática, v. 26, n. 42 B, p. 609-626, 2012.

MOURA, D. 'H. A relação entre a educação profissional e a educação básica na CONAE 2010: possibilidades e limites para a construção do novo Plano Nacional de Educação. Educação e Sociedade, n. 112, p. 875-894, 2010. 
Integrated secondary education: subsumption to the interests of the capital or crossing towards an integral human formation? Educação e Pesquisa, v. 39, n. 3, p. 705-720, 2013.

PERISSÉ, A.; GOMES, M.; NOGUEIRA, S. Revisões sistemáticas (inclusive metanálises) e diretrizes clínicas. In: GOMES, M. (Ed.). Medicina baseada em evidências: princípios e práticas. Rio de Janeiro: Reichmann \& Affonso, 2001. p.131.

PRADO, R. D.; BRANDÃO, A. A.; BRANDÃO, L. D. O. iGraf: an interactive graphical software for CSET education. Frontiers in Education Conference (FIE), 2011, 2011. IEEE. p.T1E-1-T1E-6.

ROCHADEL, W. et al. Application of mobile devices and remote experiments for physics teaching in elementary education. Global Engineering Education Conference (EDUCON), 2013 IEEE, 2013. IEEE. p.880-885.

SAMPAIO, R. F.; MANCINI, M. C. Estudos de revisão sistemática: um guia para síntese criteriosa da evidência científica. Braz. J. Phys. Ther.(Impr.), v. 11, n. 1, p. 8389, 2007.

SHIROMA, E. O.; LIMA FILHO, D. L. Trabalho docente na Educação Profissional e Tecnológica e no PROEJA. Educação \& Sociedade, v. 32, n. 116, p. 725-743, 2011.

SILVA, J. B. D. et al. Adaptation Model of Mobile Remote Experimentation for Elementary Schools. Tecnologias del Aprendizaje, IEEE Revista Iberoamericana de, v. 9, n. 1, p. 28-32, 2014.

STÊNICO, J. A.; SILVA, J. M. A. P. Educação profissional brasileira: Análises, discussões e tendências. Revista Espacios, v. 35, n. 12, 2014.

TAJRA, S. F. Informática na Educação: novas ferramentas pedagógicas para o professor na atualidade. Érica, 2011. ISBN 8536502002.

UNESCO. Diretrizes de políticas para a aprendizagem móvel. Brasil: 2014.

\section{Como referenciar este artigo}

NICOLETE, Priscila Cadorin et al. Integração Tecnológica na educação básica pública brasileira: uma análise sobre a evolução temporal e a interdisciplinaridade do tema. Revista Ibero-Americana de Estudos em Educação, Araraquara, v. 11, n. 4, p. 20642086, 2016. Disponível em: 〈http://dx.doi.org/10.21723/riaee.v11.n4.8155>. E-ISSN: 1982-5587.

Submetido em: outubro/2015

Aprovação final em: outubro/2016 